\title{
Odontoma composto em região anterior de mandíbula: relato de caso
}

\author{
Composite odontoma in the anterior region of the mandible: a case report
}

Odontoma compuesto en la región anterior de la mandíbula: informe del caso

Igor Figueiredo Pereira', Jeferson Batista Santiago ${ }^{1 *}$, Diego Dias Neves ${ }^{1}$, Olívia Augusta Araújo Dias $^{1}$, Joana de Ângelis Alves Silva ${ }^{1}$, Maria Flávia de Paiva Siqueira ${ }^{1}$, Paulo Correia Barbosa Filho², Erika Renata Carvalho Araujo', Paulina Bernardino de Andrade', Alana Cristina Santos de Araújo'.

\section{RESUMO}

Objetivo: Relatar um caso de odontoma composto localizado em região anterior de mandíbula. Detalhamento do caso: Paciente de 18 anos, gênero masculino, compareceu ao serviço de cirurgia e traumatologia buco-maxilo-facial encaminhado pelo ortodontista. Ao exame clínico, foi observado a ausência do elemento 42 e 43, com abaulamento em região anterior de mandíbula. Aos exames imaginológicos, observou-se que os elementos 42 e 43, encontravam-se inclusos associados a lesões hiperdensas semelhantes a dentes. O paciente não relatava sintomatologia. Sob anestesia geral, intubação nasotraqueal, foi realizada incisão em envelope, com descolamento mucoperiosteal total para exposição de toda a região cirúrgica. Foi realizada a osteotomia para remoção das lesões e odontossecção para remoção dos elementos inclusos. Ao final do procedimento, utilizou-se uma membrana hemostática absorvível. Pelo aspecto clínico e radiográfico característico, foi dado o diagnóstico de odontoma composto. O paciente recebeu antibióticoterapia, anti-inflamatório e analgésico. Considerações finais: Odontoma composto é uma malformação benigna, com evolução lenta. Seu tratamento consiste na excisão cirúrgica, na qual deve ser realizada a completa remoção e curetagem da lesão, a fim de promover a reabilitação precoce e/ou evitar futuras sequelas.

Palavras-chave: Odontoma, Anormalidades dentárias, Hamartoma.

\begin{abstract}
Objective: To report a case of composite odontoma located in the anterior region of the mandible. Case details: An 18-year-old male patient attended the buccomaxillofacial surgery and traumatology service referred by the orthodontist. On clinical examination, the absence of elements 42 and 43 was observed, with bulging in the anterior region of the mandible. On imaging studies, it was observed that elements 42 and 43 were included in association with hyperdense lesions similar to teeth. The patient did not report any symptoms. Under general anesthesia, nasotracheal intubation, an enveloped incision was made, with total mucoperiosteal detachment to expose the entire surgical region. Osteotomy was performed to remove the lesions and odontossection to remove the included elements. At the end of the procedure, an absorbable hemostatic membrane was used. Due to the characteristic clinical and radiographic aspect, a diagnosis of compound odontoma was made. The patient received antibiotic therapy, anti-inflammatory and analgesic. Final considerations: Composite odont oma is a benign malformation, with slow evolution. Its treatment consists of surgical excision, in which the complete removal and curettage of the lesion must be performed, in order to promote early rehabilitation and / or prevent future sequelae.
\end{abstract}

Key words: Odontoma, Tooth abnormalities, Hamartoma.

${ }^{1}$ Faculdade de Odontologia de Pernambuco (FOP-UPE), Camaragibe - PE.

${ }^{2}$ Faculdade de Ciências Médicas (FCM-UPE), Recife - PE. *E-mail: jefersonbatistas@hotmail.com

SUBMETIDO EM: 7/2020

ACEITO EM: 8/2020

PUBLICADO EM: 10/2020 


\section{RESUMEN}

Objetivo: Reportar un caso de odontoma compuesto ubicado en la región anterior de la mandíbula. Detalles del caso: Un paciente masculino de 18 años asistió al servicio de traumatología y cirugía buccomaxilofacial referido por el ortodoncista. En el examen clínico, se observó la ausencia de elementos 42 y 43 , con abultamiento en la región anterior de la mandíbula. En los estudios de imagen, se observó que los elementos 42 y 43 se incluyeron en asociación con lesiones hiperdensas similares a los dientes. El paciente no informó ningún síntoma. Bajo anestesia general, intubación nasotraqueal, se realizó una incisión envuelta, con desprendimiento mucoperióstico total para exponer toda la región quirúrgica. La osteotomía se realizó para eliminar las lesiones y la odontosección para eliminar los elementos incluidos. Al final del procedimiento, se utilizó una membrana hemostática absorbible. Debido al aspecto clínico y radiográfico característico, se realizó un diagnóstico de odontoma compuesto. El paciente recibió terapia antibiótica, antiinflamatoria y analgésica. Consideraciones finales: El odontoma compuesto es una malformación benigna, con evolución lenta. Su tratamiento consiste en la escisión quirúrgica, en la que se debe realizar la extirpación completa y el legrado de la lesión, para promover la rehabilitación temprana y / o prevenir secuelas futuras.

Palabras clave: Odontoma, Anomalías dentarias, Hamartoma.

\section{INTRODUÇÃO}

Os tumores odontogênicos possuem uma baixa prevalência, de 0,002 a $0,1 \%$, sendo o odontoma o mais comum, tendo uma incidência entre $20 \%$ a $67 \%$ de todos os tumores odontogênicos (ESWARA UMA, 2017; PREOTEASA CT e PREOTEASA E, 2018; KÄMMERER PW, et al., 2016).

Em 1867, Paul Brocain, descreveu os odontomas pela primeira vez. Foi utilizado o termo odontoma para classificar todos os tumores odontogênicos, porém atualmente este termo é muito mais específico (ESWARA UMA, 2017; SALGADO H e MESQUITA P, 2013).

Os odontomas são considerados malformações de desenvolvimento (hamartomas) ao invés de neoplasias verdadeiras. Segundo a Organização Mundial da Saúde (OMS) os odontomas podem ser classificados em dois tipos (de acordo com características radiográficas), compostos e complexos (KUNUSOTH R, et al., 2019; YADAV M, et al., 2012).

Sua prevalência excede a de todos os outros tumores odontogênicos combinados, geralmente apresentam crescimento lento e são assintomáticos, podendo ou não estar associados ao cisto dentígero (GIRISH G, et al., 2016; MATOS A, et al., 2012; SANTOS MESM, et al., 2010).

Grande parte dos odontomas são encontrados quando um paciente é avaliado radiograficamente por conta de distúrbios de erupção dentária. A maioria dos dentes não irrompidos por conta de odontomas encontramse na dentição permanente, embora esse problema também possa ser encontrado na dentição mista precoce (PILLAl A, et al., 2013).

A patogênese dos odontomas pode estar relacionada a alguns fatores, tais como: traumas na dentição primária, hiperatividade de odontoblastos, alterações em componentes genéticos responsáveis pelo desenvolvimento dentário, infecções, anomalias hereditárias como a síndrome de Gardner e hiperatividade dos odontoblastos (ESWARA UMA, 2017; IATROU I, et al., 2010; KÄMMERER PW, et al., 2016; PILLAI A, et al., 2013).

Geralmente ocorrem durante as primeiras duas décadas de vida, sendo a predileção por gênero ainda bastante discutida, porém alguns artigos apontam por uma incidência maior no gênero masculino, sendo assintomático e descoberto através de exames de rotina, e por estarem, muitas vezes, associados à falta de erupção de um dente permanente. Geralmente são encontrados em tamanhos relativamente pequenos, entretanto, se chegarem à $6 \mathrm{~cm}$ ou mais, podem levar à expansão cortical dos ossos gnáticos (BOFFANO $P$, et al., 2012; ESWARA UMA, 2017; NETO AEM e CAPELLA DL, 2011; SANTOS MESM, et al., 2010). 
Os odontomas compostos diferenciam-se dos complexos, principalmente, por serem uma malformação a qual tecidos dentários são formados em um padrão mais ordenado, ao contrário do complexo que possui um padrão menos ordenado (GIRISH G, et al., 2016). Esses odontomas compostos são formados por múltiplas estruturas semelhantes a dentes, constituídos por elementos dentários mesenquimais e epiteliais, sendo uma malformação benigna com evolução lenta, apresentando-se ainda mais comum do que o complexo (GIRISH G, et al., 2016; SANTOS MESM, et al., 2010). Bereket C, et al. (2015) analisou 69 casos desses dois tipos de odontoma e os resultados demonstraram uma clara predominância de 2,5: 1, das lesões compostas sobre odontomas complexos.

São formados pela divisão do órgão do esmalte normal em muitos outros pequenos, que dão origem às numerosas e variedades formas de dentículos, os quais estão unidos por um tecido conjuntivo fibroso, cemento ou substância óssea (NETO AEM e CAPELLA DL, 2011). Em um corte histológico, podem ser observados tecidos dentários incluindo esmalte, dentina, cemento e, em alguns casos, tecido pulpar (GIRISH G, et al., 2016). Neville BW et al. (2016) afirmam ainda, que os odontomas compostos apresentam-se, histologicamente, com múltiplas estruturas lembrando pequenos dentes unirradiculares, contidos em uma matriz fibrosa frouxa, os complexos consistem, na maioria das vezes, de dentina tubular madura.

Radiograficamente, os odontomas compostos mostram-se como uma coleção de estruturas semelhantes a dentes de variados tamanhos e formas, cercados por uma zona radiolúcida. Já os complexos, aparecem como um aglomerado de massa radiopaca bem delimitada, sendo também revestida por uma imagem radiolúcida (MATOS A, et al., 2012; SANTOS MESM, et al., 2010).

O diagnóstico geralmente se faz durante exames radiográficos de rotina (KIM KS, et al., 2019). Seu tratamento consiste na excisão cirúrgica, na qual deve ser realizada a completa remoção e curetagem da lesão, a fim de promover a reabilitação precoce e/ou evitar futuras sequelas, como por exemplo a formação de um cisto ou até mesmo a conversão em doenças malignas como o odontoameloblastoma. A recidiva é raramente reportada (NETO AEM e CAPELLA DL, 2011; SANTOS MESM, et al., 2010; KIM KS, et al., 2019). Diante disso, o objetivo do presente estudo é relatar um caso de odontoma composto, na região anterior de mandíbula, com expansão da cortical óssea.

\section{RELATO DE CASO}

Paciente B.P.A, gênero masculino, 18 anos, compareceu ao serviço de cirurgia e traumatologia bucomaxilo-facial, encaminhado pelo ortodontista para remoção de lesões e de dentes inclusos em região anterior de mandíbula, pois havia incapacidade de tracionamento destes elementos, consistindo o plano de tratamento, em alinhamento ortodôntico sem a presença dos dentes inclusos.

Ao exame clínico, foi observado, ausência do elemento 42 e 43, presença de dentes decíduos (82 e 83 ) com retenção prolongada dos mesmos, e abaulamento em região anterior de mandíbula. $O$ paciente não relatava sintomatologia dolorosa ou alteração sistêmica. Ao exame tomográfico (reconstrução panorâmica), foi observado que os elementos 42 e 43 encontravam-se inclusos, associados a múltiplas lesões hiperdensas semelhantes a dentes (Figura 1).

Figura 1 - Tomografia computadorizada (reconstrução panorâmica) apresentando imagens hiperdensas em região anterior de mandíbula.

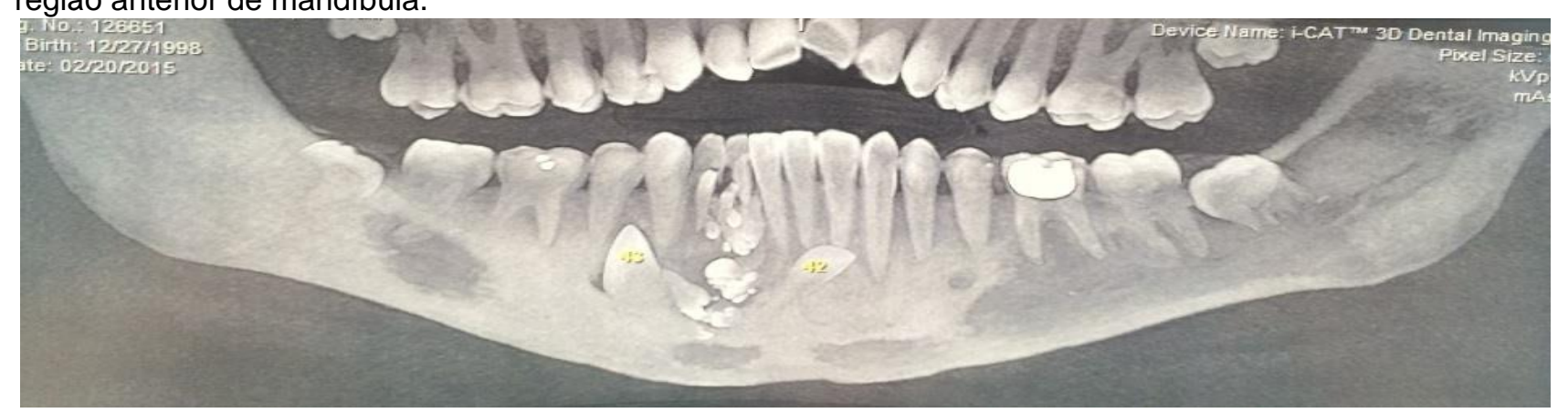

Fonte: PEREIRA IF, et al., 2020 
Sob anestesia geral, intubação nasotraqueal, foi realizada a remoção dos dentes decíduos, e logo em seguida a incisão em envelope, com descolamento mucoperiosteal total, estendida do elemento 46 até 0 dente 35 para exposição de toda a região vestibular da mandíbula (Figura 2).

Figura 2 - Aspecto transoperatório da incisão em envelope, com descolamento mucoperiosteal total.

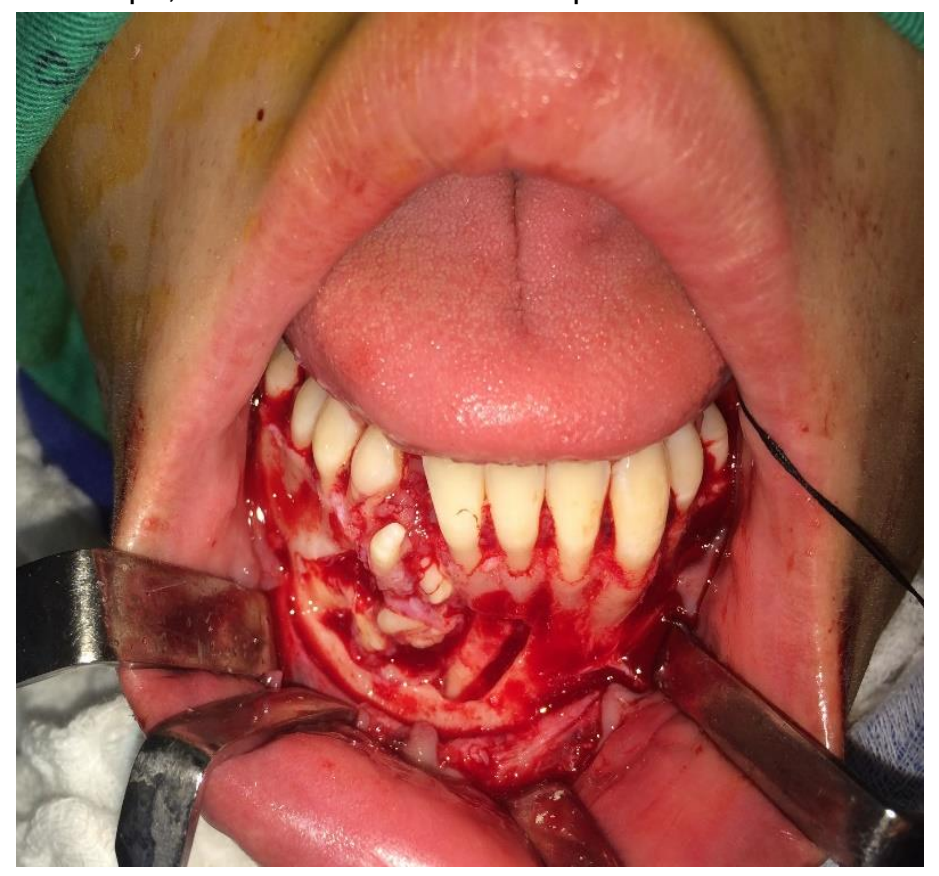

Fonte: PEREIRA IF, et al., 2020.

Foi realizada a osteotomia para remoção das lesões (sete dentículos) e odontosecção para remoção dos elementos dentários inclusos, buscando a maior preservação óssea possível (Figura 3).

Figura 3 - Odontomas compostos removidos.

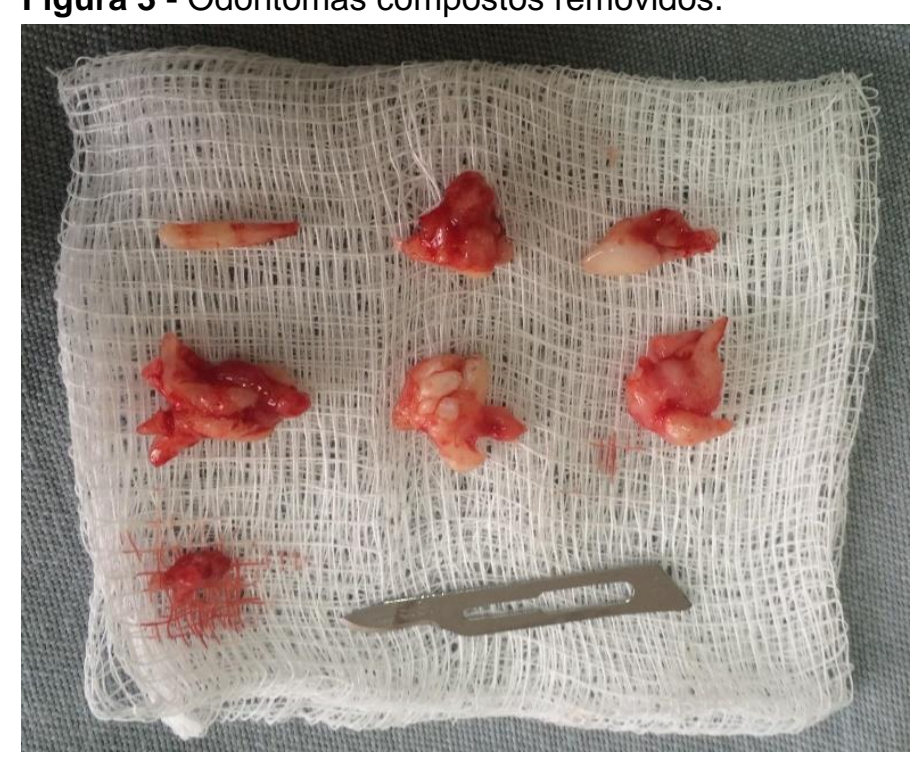

Fonte: PEREIRA IF, et al., 2020.

Ao final do procedimento, foi utilizada uma membrana hemostática absorvível, Surgicel® (Johnson e Johnson Medical, Arlington, TX), para formação de um arcabouço, com o objetivo de favorecer a neoformação óssea (Figura 4A). O fechamento da ferida foi realizado por primeira intenção (Figura 4B). 
Figura 4 - A) Aspecto transoperatório com aplicação do Surgice|®. B) Fechamento da ferida cirúrgica.

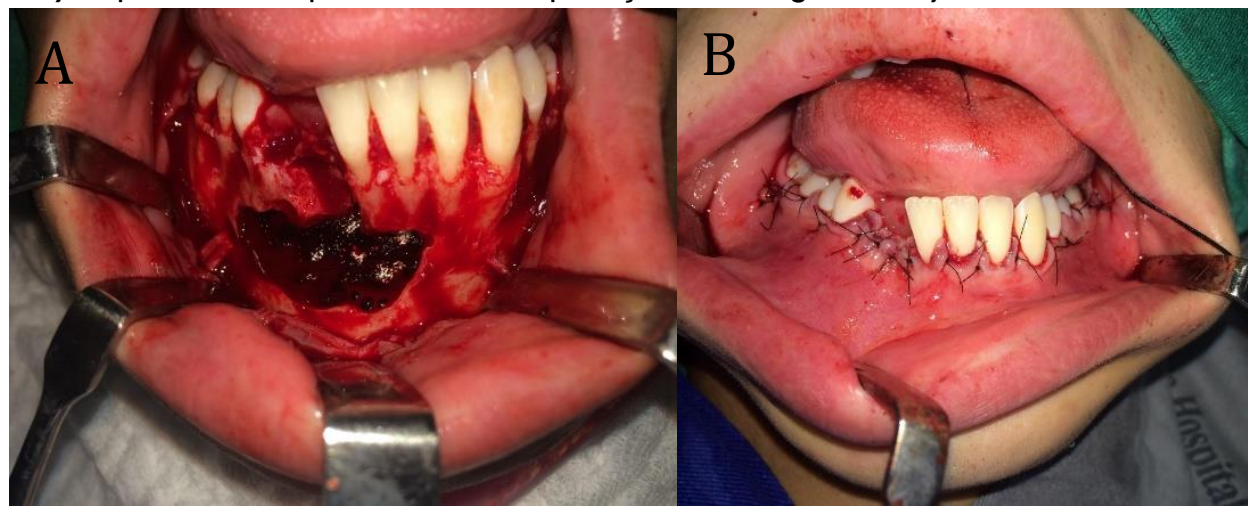

Fonte: PEREIRA IF, et al., 2020.

No dia seguinte ao procedimento, o paciente se encontrava estável, sem queixas, com discreto edema, compatível com o ato cirúrgico, portanto, recebeu alta hospitalar e foi medicado com antibiótico: (amoxicilina $500 \mathrm{mg}, 1$ cápsula de 8 em 8 horas durante 7 dias), anti-inflamatório (nimesulida 100mg, 1 comprimido de 12 em 12 horas por 3 dias) e analgésico (dipirona 500mg, 1 comprimido de 6 em 6 horas por 3 dias).

\section{DISCUSSÃO}

Este caso relata uma lesão encontrada em um paciente do sexo masculino de 18 anos, idade que coincide com a faixa etária reportada na literatura, que mostra uma maior incidência na segunda e terceira década de vida (GIRISH G, et al., 2016; SILVA DJS, et al., 2015), no entanto podem ser observados em qualquer idade (LEVI e ARDILA, 2019; SALGADO H e MESQUITA P, 2013). Segundo Poli P, et al. (2019) o odontoma é mais prevalente no gênero masculino, porém alguns autores afirmam não ter predileção por sexo (AN $S$, et al., 2012; TEKKESIN MS, et al., 2012).

Devido a essas malformações serem patogenicamente diferentes, é compreendido que também haja diferenças na incidência com relação a idade do paciente e localização da lesão. Enquanto os odontomas complexos são lesões em estágio terminal de um hamartoma, serão encontradas mais frequentemente nos idosos e em região posterior mandíbular, já os compostos formam-se a partir da hiperatividade local da lâmina dentária, que é muito comum em pessoas jovens (ESWARA UMA, 2017; GONZÁLEZ-ALVA P, et al., 2011).

Essas informações corroboram com o presente caso, uma vez que o paciente é jovem, e a atividade da lâmina dentária é maior entre esses, porém neste caso não se sabe de fato qual foi o fator causador da hiperatividade da lâmina dentária, pois ele relatou não haver história familiar relacionada a doença, nem trauma ou infecção prévia.

Os odontomas causam transtornos oclusais, interferindo no processo de erupção dentária, deslocam e ocasionam má formação dos dentes vizinhos e, em alguns casos, erupção ectópica. Além disso podem estar relacionados com casos de assimetrias faciais, diastemas persistentes e divergências do longo eixo dos dentes. O odontoma composto é comumente encontrado próximo a dentes e pode estar associado tanto ao atraso na erupção como também a má posição dentária (JAEGER $\mathrm{F}$, et al., 2012; PREOTEASA CT e PREOTEASA E, 2018).

Neste caso, os diversos dentículos estavam adjacentes aos dentes 42 e 43 inclusos, aos quais podemos associar como sendo o principal fator causador da retenção dos mesmos, e consequentemente provocando uma retenção prolongada dos dentes decíduos 82 e 83 presentes no arco (Figura 1).

Os odontomas são tumores de lenta evolução, assintomáticos e na maioria dos casos de pequeno volume (SANTOS MESM, et al., 2010). Segundo Freire AL, et al. (2018) a sintomatologia dolorosa pode estar presente em alguns casos: o odontoma, quando grande, expande a cortical óssea podendo comprimir estruturas anatômicas importantes, como as estruturas nervosas. No presente caso, os odontomas foram de pequenas proporções, porém provocaram expansão óssea, ainda que sem sintomatologia dolorosa. 
Os odontomas também podem ser classificados clinicamente como intraósseo ou extraósseo. Os intraósseos ocorrem dentro da maxila ou mandíbula, e há a possibilidade de entrarem em erupção na cavidade oral, inclusive infecções recorrentes da erupção intra oral já foram relatadas. Já o extraósseos são os odontomas periféricos oriundos dos tecidos moles, e são raros (KÄMMERER PW, et al., 2016; KIM KS, et al., 2019). O paciente deste caso possuía um odontoma intraósseo que não erupcionou na cavidade oral.

Alguns estudos mostraram que a localização mais comum do odontoma composto é a região anterior da maxila (GIRISH G, et al., 2016; ESWARA UMA, 2017; AN S, et al., 2012). No presente caso os odontomas localizavam-se na região anterior de mandíbula, o que contrapõe a maioria dos achados clínicos. Apesar de ser incomum existem alguns relatos da presença do odontoma composto na região anterior de mandíbula. (DE SOUSA-NETO, et al., 2019; DO NASCIMENTO, et al., 2019). Outro estudo mostrou uma maior frequência dos odontomas no lado direito da mandíbula, o que também ocorreu no presente caso (SALGADO $\mathrm{H}$ e MESQUITA P, 2013).

As características radiográficas dos odontomas compostos são caracterizadas por dentículos de tamanhos variados compactados numa massa envolta por um halo radiolúcido bem delimitado, semelhante ao achado radiográfico da lesão em questão, que se apresentou bem característica (NEVILLE BW et al., 2016). Pelo aspecto clínico e radiográfico característico, o diagnóstico geralmente é preciso, sendo o exame histopatológico apenas para ratificar os achados (GIRISH G, et al., 2016).

Esses tumores apresentam um alto nível de organização dos tecidos internos (PREOTEASA CT e PREOTEASA E, 2018; ESWARA UMA, 2017). O material obtido na exérese é constituído por múltiplos dentículos, o que coincide com as características presentes na literatura para odontomas compostos, dando suporte ao diagnóstico estabelecido. Pôde ser notado que os dentículos apresentavam muitas estruturas calcificadas com tamanhos e formatos variados, organizados em conglomerados com a superfície irregular, e alguns eram semelhantes a micro dentes. (PREOTEASA CT e PREOTEASA E, 2018).

O tratamento foi conservador, realizando-se apenas a excisão cirúrgica dos dentículos e dos dentes inclusos envolvidos, sem a necessidade de realizar osteotomia periférica ou ressecção pelo fato da recidiva ser bastante rara. Esta conduta vai de acordo com a literatura, que prova um grande número de sucessos nos casos que foram realizados esse tipo de tratamento (GIRISH G, et al., 2016; POLI PP, et al., 2019; WANDERLEY AEC, et al., 2019).

Ainda não existe um consenso sobre uma conduta ideal para dentes impactados associados ao odontoma, porém um estudo indicou que devem ser preservados quando possível (KÄMMERER PW, et al., 2016; AN S, et al., 2012). Optou-se pelo não aproveitamento dos dentes inclusos envolvidos, por conta da orientação e planejamento ortodôntico, que informou a não necessidade destes, para o processo de alinhamento da arcada inferior. A conduta de tracionamento ortodôntico nesse caso seria mais complexa, sendo o realinhamento da arcada com os dentes remanescentes uma opção mais simples e viável.

Este trabalho relatou um caso de odontoma composto que se desenvolveu na região anterior da mandíbula. O mesmo causou abaulamento na região e estava associado a impactação de dentes permanentes e atraso na esfoliação de decíduos. O tratamento de escolha consistiu na excisão cirúrgica, com osteotomia e odontosecção, para remover toda a lesão, preservando maior quantidade óssea possível, a fim de promover a reabilitação e evitar futuras sequelas. De acordo com o planejamento ortodôntico e o espaço disponível na arcada dentária do paciente, os dentes não necessitaram de tracionamento ortodôntico.

\section{REFERÊNCIAS}

1. AN S, et al. Odontoma: a retrospective study of 73 cases. Imaging Science in Dentistry, 2012; 42(2): 77-81.

2. BEREKET C, et al. Complex and compound odontomas: Analysis of 69 cases and a rare case of erupted compound odontoma. Nigerian journal of clinical practice, 2015; 18(6): 726-730.

3. BOFFANO P, et al. Complex and compound odontomas. Journal of Craniofacial Surgery, 2012; 23(3): 685-688.

4. DE SOUSA-NETO SS, et al. Odontoma composto em adulto e sua complexidade de tratamento: relato de caso clínico. Revista Odontológica do Brasil Central, 2019; 28(87): 266-299.

5. DO NASCIMENTO FB, et al. Dente impactado associado a odontoma composto: relato de caso clínico. Revista uningá, 2019; 56(7): 180-186. 
6. ESWARA UMA, et al. Compound odontoma in anterior mandible-a case report. The Malaysian Journal of Medical Sciences, 2017; 24(3): 92.

7. FREIRE AL, et al. Odontoma composto em paciente pediátrico: relato de caso. Revista de Iniciação Científica em Odontologia, 2018; 16(3): 91-99.

8. GIRISH G, et al. Compound composite odontoma. Journal of Oral and Maxillofacial Pathology: JOMFP, 2016; 20(1): 162.

9. GONZÁLEZ-ALVA P, et al. Podoplanin expression in odontomas: clinicopathological study and immunohistochemical analysis of 86 cases. Journal of oral science, $2011 ; 53(1): 67-75$.

10. IATROU I, et al. A retrospective analysis of the characteristics, treatment and follow-up of 26 odontomas in Greek children. Journal of oral Science, 2010; 52(3): 439-447.

11. JAEGER F, et al. Odontoma composto-relato de caso clínico. Revista Portuguesa de Estomatologia, Medicina Dentária e Cirurgia Maxilofacial, 2012; 53(4): 252-257.

12. KÄMMERER PW, et al. Clinical parameter of odontoma with special emphasis on treatment of impacted teeth-a retrospective multicentre study and literature review. Clinical oral investigations, 2016; 20(7): 1827-1835.

13. KIM KS, et al. Incidentally detected odontoma within a dentigerous cyst. Archives of craniofacial surgery, 2019; 20(1): 62.

14. KUNUSOTH R, et al. Compound odontoma in the anterior maxilla. Oncology and Radiotherapy, 2019; 1(46): 43-45.

15. LEVI-DUQUE F, ARDILA CM. Association between odontoma size, age and gender: Multivariate analysis of retrospective data. Journal of clinical and experimental dentistry, 2019;11(8): 701.

16. MATOS A, et al. Odontoma composto em região posterior de mandíbula: relato de caso. Oral Science, 2012; 4(2): 5458.

17. NETO AEM, Capella DL. Tratamento conservador de grande odontoma complexo em mandíbula. Revista da Faculdade de Odontologia Universidade de Passo Fundo, 2011; 16(3): 317-321.

18. NEVILLE BW, et al. Patologia Oral e Maxilofacial. 4를 ed. Rio de Janeiro: Elsevier, 2016; 928p.

19. PILLAI A, et al. A complex odontoma of the anterior maxilla associated with an erupting canine. Case Reports, 2013; 2013: bcr2013200684.

20. PIRES LD, et al. Odontoma: estado da arte e relato de caso clínico. Stomatos, 2007; 13(24): 21-9.

21. POLI PP, et al. Anxiolysis in the surgical management of a compound odontoma in a pediatric patient. Case Reports in Dentistry, 2019; 2019: 1-4.

22. PREOTEASA CT, PREOTEASA E. Compound odontoma-morphology, clinical findings and treatment. Case report. Romanian Journal of Morphology and Embryology, 2018; 59(3): 997-1000.

23. SALGADO H, MESQUITA P. Compound odontoma-case report. Revista Portuguesa de Estomatologia, Medicina Dentária e Cirurgia Maxilofacial, 2013; 54(3): 161-165.

24. SANTOS MESM, et al. Odontoma como fator de retenção dentária: relato de casos clínicos. Revista de Cirurgia e Traumatologia Buco-maxilo-facial, 2010; 10(2): 25-30.

25. SILVA DJS, et al. Estudo Clínico-Patológico de Odontomas Diagnosticados no Laboratório de Patologia Bucal da Faculdade de Odontologia de Pernambuco-FOP. Revista de Cirurgia e Traumatologia Buco-maxilo-facial, 2015; 15(4): 31-36.

26. TEKKESIN MS, et al. Clinical and histopathological investigation of odontomas: review of the literature and presentation of 160 cases. Journal of Oral and Maxillofacial Surgery, 2012; 70(6): 1358-1361.

27. WANDERLEY AEC, et al. Odontoma composto como fator de impactação dentária: Relato de caso. Revista Eletrônica Acervo Saúde, 2019; 34: 1794-1794.

28. YADAV M, et al. Compound Odontoma. Contemporary Clinical Dentistry, 2012; 3: 13-15. 